\title{
Inflamed fat: what starts the fire?
}

\author{
Jaap G. Neels and Jerrold M. Olefsky \\ Department of Medicine, Division of Endocrinology and Metabolism, UCSD, La Jolla, California, USA.
}

\begin{abstract}
Obesity is associated with increased macrophage infiltration of adipose tissue, and these macrophages may be an important component of the chronic inflammatory response playing a crucial role in the development of insulin resistance. This prompts the question as to how macrophages infiltrate obese adipose tissue. In this issue of the JCI, Weisberg et al. show the importance of $\mathrm{C}-\mathrm{C}$ motif chemokine receptor 2 (CCR2) in macrophage recruitment to adipose tissue and the development of obesity and its complications (see the related article beginning on page 115).
\end{abstract}

\section{Insulin resistance, inflammation, and macrophages}

Insulin resistance is a characteristic feature of most patients with type 2 diabetes mellitus, simple obesity, polycystic ovarian syndrome, and impaired glucose tolerance, as well as other disorders (1). There are also a number of other manifestations associated with insulin resistance, and this constellation of abnormalities is generally described by the term "syndrome X" or "metabolic syndrome" (1). Decreased insulin sensitivity is the central feature of this syndrome, and other components include obesity, hypertension, dyslipidemia, and hyperuricemia (1). In recent years, the general concept has emerged that chronic activation of the proinflammatory pathway can be a mechanism for insulin resistance (2). Various studies have implicated chronic activation of the NF- $\kappa \mathrm{B}$ proinflammatory pathway and/or JNK1 as underlying mechanisms, and most of these studies have focused on activation of this pathway within insulin target tissues (adipose, liver, and muscle) as an etiologic mechanism (2). The adipocyte has emerged as an important player in this process, since this cell type can be the source of a large number of cytokines/chemokines (TNF- $\alpha$, IL-6, IL-1, monocyte chemoattractant protein-1, etc.) and adipokines (leptin, adiponectin, and resistin), and all of these molecules can exert potential negative or positive effects on insulin sensitivity in an endocrine or paracrine fashion (Figure 1A) (3). While

Nonstandard abbreviations used: ATM, adipose tissue macrophage; CCL2, C-C motif chemokine ligand 2; CCR2, C-C motif chemokine receptor 2; HFD, high-fat diet.

Conflict of interest: The authors have declared that no conflict of interest exists.

Citation for this article: J. Clin. Invest. 116:33-35 (2006). doi:10.1172/JCI27280. there is no question that the inflammatory pathway becomes activated in adipose tissue in various insulin-resistant states, and that this has deleterious effects on insulin action in adipocytes, the total contribution of adipose tissue as a systemic source of these circulating cytokines/chemokines is still unclear. In this regard, recent attention has focused on the potential role of macrophages in this process (4). Thus, Weisberg et al. and Xu et al. have shown that, in obesity, adipose tissue contains an increased number of resident macrophages and that, in some circumstances, macrophages can constitute up to $40 \%$ of the cell population within an adipose tissue depot $(5,6)$. Macrophages are obviously a potential source of secreted proinflammatory factors, and these correlative data have led to the concept that macrophages can directly influence adipocyte biology and systemic insulin resistance. Taking this story further, Arkan et al. have recently presented evidence indicating the causal role of the macrophage in leading to insulin resistance in mice (7). They disabled the inflammatory pathway within macrophages by creating myeloid-specific inbibitor of $N F-\kappa B$ kinase $\beta$ $(I K K \beta) \mathrm{KO}$ animals. When fed a high-fat diet (HFD), the macrophage-specific IKK $\beta$ $\mathrm{KO}$ mice were relatively protected from glucose intolerance and hyperinsulinemia, and euglycemic clamp studies showed a global improvement in insulin sensitivity in all insulin target tissues. These studies are consistent with the interpretation that the macrophage is an important, and potentially initiating, cell type in the process of inflammation-induced insulin resistance.

\section{Role of CCR2}

The mechanism of macrophage recruitment to adipose tissue has not been defined in detail, but it presumably involves increased secretion of chemotactic molecules by adipose tissue. One such candidate is $\mathrm{C}-\mathrm{C}$ motif chemokine ligand 2 (CCL2; also known as monocyte chemoattractant protein-1). CCL2 is a member of the C-C motif chemokine family and is a major ligand for $\mathrm{C}-\mathrm{C}$ motif chemokine receptor 2 (CCR2) (8). CCL2 and its receptor CCR2 are crucial for monocyte/macrophage recruitment in several inflammatory models, and CCL2 is highly expressed in the adipose tissue of obese subjects and rodents (8-10). Furthermore, CCR 2 is expressed by adipocytes, and incubation of 3T3-L1 adipocytes with CCL2 leads to a state of cellular insulin resistance, as manifested by decreased insulin-stimulated glucose uptake (Figure 1A) (9). Addition of CCL2 to differentiated adipocytes in vitro decreases expression of several adipogenic genes, suggesting a role for CCL2 and CCR2 in adipogenesis (9).

In this issue of the JCI, Weisberg et al. (11) report their studies assessing the role of CCR2 in macrophage recruitment to adipose tissue and the development of obesity and insulin resistance. They show that, in HFD-fed mice, CCR2 deficiency attenuated adipose tissue macrophage (ATM) accumulation, adipose tissue inflammation, and systemic insulin resistance. In mice with preexisting obesity, short-term pharmacologic antagonism of CCR2 reduced ATM content and improved insulin sensitivity. Of interest, they also found that $\mathrm{KO}$ of CCR2 had direct effects on the development of adiposity, at least in a subset of animals fed an HFD. In CCR2 KO animals fed chow diets, no differences in insulin sensitivity or adipose mass or function were noted. When mice were placed on an HFD, food intake was decreased in the KO mice, and, while these animals became obese, the increased adiposity was less than that observed in the WT animals. Since CCR2 is widely expressed, including in the CNS (12), the authors postulate a possible central effect of CCR2 deficiency on eating behavior. Interestingly, they found that adipocyte cell size was increased in the $\mathrm{KO}$ animals, implying that CCR2 deficiency, in some way, results in a decrease in adipocyte cell number, which might be related to the proposed function of the CCL2/CCR2 system in adi- 
A

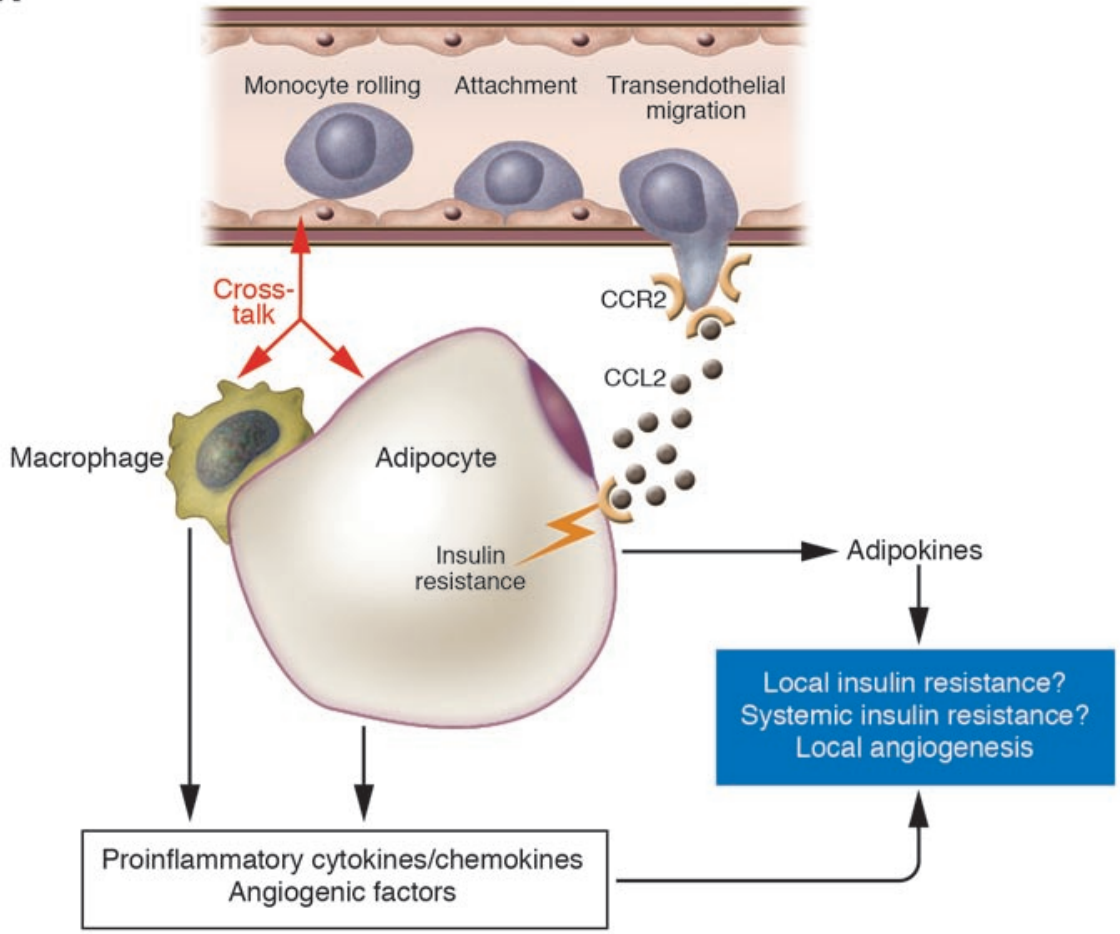

B

Lean AT

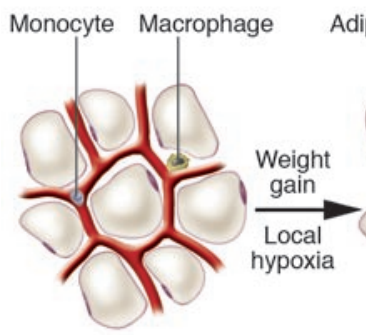

Expanding AT

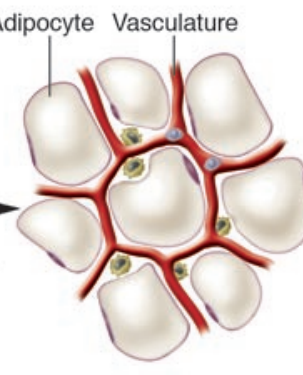

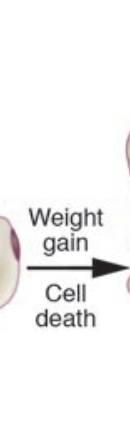

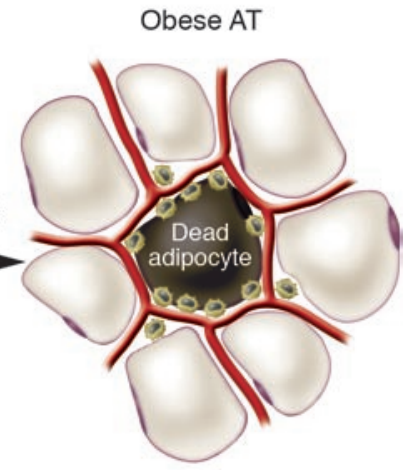

Figure 1

Macrophage itinerary in adipose tissue infiltration. (A) Macrophage infiltration occurs after initial rolling and attachment of monocytes to activated endothelial cells. These monocytes then extravasate through the endothelial cell layer and differentiate into macrophages. In this issue of the $\mathrm{JCl}$, Weisberg et al. show that CCL2 and its receptor CCR2 play an important role in macrophage chemotaxis (11). Cross-talk among adipocytes, macrophages, and endothelial cells may aggravate the inflammatory state, resulting in increased secretion of proinflammatory cytokines/chemokines, adipokines, and angiogenic factors. These factors could cause local and/or systemic insulin resistance in a paracrine and/or endocrine fashion, respectively, and might also induce local angiogenesis. (B) Adipose tissue (AT) expansion during weight gain leads to recruitment of macrophages through a variety of signals, which may include local hypoxia. These macrophages predominantly localize around dead adipocytes.

pogenesis (9). Although some of the CCR2 $\mathrm{KO}$ animals gained less weight on an HFD than WT mice, the authors were careful to control for this point by studying groups of WT and KO animals matched for adiposity. In this analysis, they clearly showed that the metabolic effects of CCR2 deficiency did not simply depend on the magnitude of obesity. When CCR2-deficient mice were weight-matched with WT controls, they still showed enhanced insulin sensitivity and decreased numbers of ATMs, as well as the other features of the phenotype. In addition, when mice were treated with a CCR2 inhibi- tor, they did not lose weight compared with control animals and yet still showed amelioration of insulin resistance.

This study (11) provides new and important evidence supporting the general view that increased adipocyte-derived CCL2 is a chemoattractant that participates in the process of macrophage recruitment into adipose tissue (Figure 1A), and suggesting that, when this process is disrupted by CCR $2 \mathrm{KO}$ or treatment with a CCR2 inhibitor, ATM content decreases, the adipose tissue inflammatory response is attenuated, and systemic insulin sensitivity improves. It should be noted, however, that different results were obtained in a recent study on CCR2 KO mice ingesting an HFD (13). In the study by Chen et al., CCR2 KO animals fed an HFD did not show any differences in weight gain compared with controls, nor were there any differences in ATM accumulation or circulating basal glucose and insulin levels. Although these studies show different results, there were also some important differences in experimental design. The period of HFD feeding was shorter in the study by Chen et al. compared with the feeding period studied by Weisberg et al. (11), and the CCR2 KO animals examined by $\mathrm{Chen}$ et al. were bred on a $D B A 1 / J$ background instead of the C57BL/6J background used by Weisberg et al. It is well known that genetic background differences can have a major effect on metabolic phenotypes, and it is possible that these background differences extend to the effects of CCR2 depletion.

The study by Weisberg et al. (11) demonstrates the importance of CCR2 for ATM infiltration, but since CCR2 deletion did not normalize macrophage content or inflammatory gene expression, additional mechanisms must be involved in ATM recruitment and retention, as suggested by the authors. Several interesting questions remain unanswered in these studies. Through what cell type(s) does the CCR2 deficiency exert its effects? Most likely, deletion of macrophage CCR2 is responsible for the decrease in ATM content, and possibly the other phenotypic effects. However, CCR2 is also expressed in adipocytes, and since CCL2 can directly cause adipocyte insulin resistance (Figure 1A) (9), the contribution of CCR 2 deficiency in adipocytes to the overall phenotype remains to be determined. Such questions could be addressed by studies of tissue-specific CCR2 $\mathrm{KO}$ animals, or by generation of chimeric animals through transplantation of bone 
marrow from CCR2 KO animals into WT mice. Additionally, what is the mechanism of the enhanced systemic insulin sensitivity observed in the CCR2 KO animals? Interestingly, adiponectin can lead to increased insulin sensitivity, but although adiponectin levels were increased in the CCR2 KO animals, levels did not change in the CCR2 antagonist-treated animals, suggesting that the increase in adiponectin was not the major cause of reduced insulin resistance with CCR2 deficiency. Furthermore, treatment with a CCR2 antagonist led to enhanced insulin sensitivity but did not change adipose tissue inflammatory gene expression, raising the question of whether activation of the inflammatory pathway within adipocytes is causally related to insulin resistance, or simply a secondary reactive event.

\section{Therapeutic implications}

Our current understanding would suggest that treatments that prevent macrophage infiltration into obese adipose tissue will have beneficial effects on the inflammatory response and the abnormal metabolic state. In pursuing these therapeutic goals, it should be kept in mind that there are normal biologic functions performed by ATMs. For example, a recent study showed that more than $90 \%$ of ATMs are located surrounding dead adipocytes (Figure 1B), suggesting that one of their normal functions is to clear necrotic debris from adipose tissue, similar to the well-known effects of macrophages in other inflammatory states (14). Other studies have indicated that local tissue microhypoxia might play a role in chemotaxis and retention of macrophages in expanding adipose tissue depots (Figure 1B) (15). Adipogenesis and angiogenesis are tightly linked during fat mass development $(16,17)$, and, like tumor growth, excessive growth of adipose tissue during the development of obesity depends on the formation of new blood vessels for the supply of oxygen and nutrients (18-20). Since chemokines such as CCL2 can also act as angiogenic factors (21), it is tempting to speculate that ATMs are involved in the neovascularization that takes place in expanding adipose tissue (Figure 1A). In this event, inhibition of macrophage infiltration might affect adipose tissue expansion by interfering with angiogenesis.

\section{Future perspectives}

Although a growing number of studies convincingly show that the ATM content is increased in obese rodents, as well as humans, it is still not clear whether ATM content and insulin resistance are causally related. We still lack definitive evidence that the presence of ATMs causes insulin resistance. It remains possible that ATMs are a marker rather than a cause of insulin resistance, and perhaps macrophages impair systemic insulin sensitivity through some other mechanism. Is the systemic insulin resistance due to secretion of soluble factors by macrophages that act locally (paracrine effects), or systemically (endocrine effects), or due to paracrine effects of macrophages that cause adipose tissue to release factors that cause the decrease in insulin sensitivity? In this respect, it would be of interest to study whether there is increased macrophage content, or localized secretion of macrophage-derived factors, in other insulin target tissues such as liver and muscle.

What causes the increase in expression of chemotactic factors, such as CCL2, that results in attraction of macrophages? Do these signals come from adipocytes or from resident macrophages or endothelial cells within adipose tissue? Does the first influx of macrophages cause subsequent attraction of more macrophages? If so, is this due to the release of chemoattractants by the macrophages themselves or increased production of these factors by adipocytes or other cells through paracrine actions of the macrophages? The interaction of macrophages with adipocytes and other insulin target tissues will clearly be the subject of intense future investigations.

Address correspondence to: Jerrold M. Olefsky, Department of Medicine, Division of Endocrinology and Metabolism, University of California, San Diego, 9500 Gilman Drive, SCRB 225, La Jolla, California 92093-0673, USA. Phone: (858) 534-6651; Fax: (858) 534-6653; E-mail: jolefsky@ucsd.edu.
1. Eckel, R.H., Grundy, S.M., and Zimmet, P.Z. 2005. The metabolic syndrome. Lancet. 365:1415-1428.

2. Wellen, K.E., and Hotamisligil, G.S. 2005. Inflammation, stress, and diabetes. J. Clin. Invest. 115:1111-1119. doi:10.1172/JCI200525102.

3. Rajala, M.W., and Scherer, P.E. 2003. Minireview. The adipocyte: at the crossroads of energy homeostasis, inflammation, and atherosclerosis. Endocrinology. 144:3765-3773.

4. Bouloumie, A., et al. 2005. Role of macrophage tissue infiltration in metabolic diseases. Curr. Opin. Clin. Nutr. Metab. Care. 8:347-354.

5. Weisberg, S.P., et al. 2003. Obesity is associated with macrophage accumulation in adipose tissue. J. Clin. Invest. 112:1796-1808. doi:10.1172/ JCI200319246.

6. Xu, H., et al. 2003. Chronic inflammation in fat plays a crucial role in the development of obesity-related insulin resistance. J. Clin. Invest. 112:1821-1830. doi:10.1172/JCI200319451.

7. Arkan, M.C., et al. 2005. IKK-beta links inflammation to obesity-induced insulin resistance. Nat. Med. 11:191-198.

8. Charo, I.F., and Taubman, M.B. 2004. Chemokines in the pathogenesis of vascular disease. Circ. Res. 95:858-866.

9. Sartipy, P., and Loskutoff, D.J. 2003. Monocyte chemoattractant protein 1 in obesity and insulin resistance. Proc. Natl. Acad. Sci.U. S. A. 100:7265-7270.

10. Bruun, J.M., Lihn, A.S., Pedersen, S.B., and Richelsen, B. 2005. Monocyte chemoattractant protein-1 release is higher in visceral than subcutaneous human adipose tissue (AT): implication of macrophages resident in the AT. J. Clin. Endocrinol. Metab. 90:2282-2289.

11. Weisberg, S.P., et al. 2006. CCR2 modulates inflammatory and metabolic effects of high-fat feeding. J. Clin. Invest. 116:115-124. doi:10.1172/JCI24335.

12. Mahad, D.J., and Ransohoff, R.M. 2003. The role of MCP-1 (CCL2) and CCR2 in multiple sclerosis and experimental autoimmune encephalomyelitis (EAE). Semin. Immunol. 15:23-32.

13. Chen, A., et al. 2005. Diet induction of monocyte chemoattractant protein- 1 and its impact on obesity. Obes. Res. 13:1311-1320.

14. Cinti, S., et al. 2005. Adipocyte death defines macrophage localization and function in adipose tissue of obese mice and humans. J. Lipid Res. 46:2347-2355.

15. Cancello, R., et al. 2005. Reduction of macrophage infiltration and chemoattractant gene expression changes in white adipose tissue of morbidly obese subjects after surgery-induced weight loss. Diabetes. 54:2277-2286.

16. Fukumura, D., et al. 2003. Paracrine regulation of angiogenesis and adipocyte differentiation during in vivo adipogenesis. Circ. Res. 93:E88-E97.

17. Neels, J.G., Thinnes, T., and Loskutoff, D.J. 2004. Angiogenesis in an in vivo model of adipose tissue development. FASEB J. 18:983-985.

18. Rupnick, M.A., et al. 2002. Adipose tissue mass can be regulated through the vasculature. Proc. Natl. Acad. Sci.U. S. A. 99:10730-10735.

19. Brakenhielm, E., et al. 2004. Angiogenesis inhibitor, TNP-470, prevents diet-induced and genetic obesity in mice. Circ. Res. 94:1579-1588.

20. Hausman, G.J., and Richardson, R.L. 2004. Adipose tissue angiogenesis. J. Anim. Sci. 82:925-934.

21. Bernardini, G., et al. 2003. Analysis of the role of chemokines in angiogenesis. J. Immunol. Methods. 273:83-101. 